\title{
The effects of dictionary and lexicon to morphological analysis
}

\begin{abstract}
Dictionary or lexicon is one of the key components for morphological analyzers, except to purely rule-based tools. However, only few studies had been performed to evaluate the effects of using dictionary or lexicon for Malay morphological analysis. In this paper, we report the result of such evaluation on our morphological analyzer called MALIM, based on auto-expansion simulation. We found that lexicon improves the performance of the analyzer in term of correctness, and also the quality of the output.
\end{abstract}

Keyword: Dictionary; Lexicon; Malay language; MALIM; Morphological analyzer 